mutation was identified in all 4 alleles in the two isolates with azithromycin MICs of $>=256 \mu \mathrm{g} / \mathrm{ml}$.

Conclusions With reducing susceptibility of cephalosporins as treatment for gonorrhoea, azithromycin may be relied on more to treat gonorrhoea. Rapid molecular detection of antimicrobial susceptibilities in N. gonorrhoeae is important to prevent the spread of untreatable multidrug resistant gonorrhoea.

\section{P3.279 FROM THE GROUND UP: BUILDING NATIONAL SURVEILLANCE OF ANTIMICROBIAL RESISTANCE IN NEISSERIA GONORRHOEAE IN CANADA}

\author{
doi:10.1136/sextrans-2013-051184.0735
}

'S L Totten, ${ }^{2}$ I Martin, ' $\mathbf{J}$ Njihia, 'T Atwood, 'C Archibald. 'Public Health Agency of Canada, Ottawa, ON, Canada, ${ }^{2}$ Public Health Agency of Canada, Winnipeg, MB, Canada

Background While there is evidence that gonococcal antimicrobial resistance (GC AMR) is increasing in parts of Canada, a national, standardised surveillance system does not currently exist to confirm these suspicions or identify the risks associated with acquiring a resistant GC infection.

Methods Currently, laboratory-based surveillance of GC AMR is standard practise for all positive gonorrhoea isolates tested by culture in Canada. Nine out of 13 provinces/territories employ culture for a proportion of the total gonorrhoea tests done in their jurisdictions (typically conducted by local/regional laboratories). Variation in methods at the provincial/territorial (P/T) level and limited epidemiologic data on resistant GC isolates limits national level surveillance.

To address gaps in current systems, a national protocol for GC AMR has been developed and approved by the Health CanadaPublic Health Agency of Canada Research Ethics Board, and recruitment of $\mathrm{P} / \mathrm{T}$ health authorities is in progress. Due to $\mathrm{P} / \mathrm{T}$ variations in public health legislation and health care practises, recruitment has necessitated innovative solutions to address the individual needs of jurisdictions while ensuring the coherence and comparability of the resulting data.

Results In 2011, the proportion of GC isolates resistant to azithromycin, penicillin, erythromycin, ciprofloxacin and tetracycline was $0.4 \%, 22.2 \%, 26.6 \%, 29.3 \%$, and $29.4 \%$, respectively. Enhanced surveillance in two jurisdictions is expected to commence in 2013. Although slightly different mechanisms are being used to address provincial needs, efforts are being made to ensure that resulting data are consistent and adhere to the national protocol.

Conclusion In Canada, surveillance of GC AMR is challenged by variations in practise and legislation at the $\mathrm{P} / \mathrm{T}$ level and competing priorities at all levels of government. Through collaboration with public health partners, progress is being made in obtaining data for analysis of national-level trends to assess risk factors associated with GC AMR and guide treatment recommendations.

\section{P3.280 GENTAMICIN SUSCEPTIBILITY PROFILE OF NEISSERIA GONORRHOEAE CLINICAL STRAINS ISOLATED IN BILBAO (SPAIN) DURING 2011 AND 2012}

doi:10.1136/sextrans-2013-051184.0736

M Nieto, G Ezpeleta, V Esteban, J Alava, M Camara, J Lopez de Munain, R Cisterna. Basurto University Hospital, Bilbao, Spain

Background Antimicrobial therapy is essential to control Neisseria gonorrhoeae (NG). The appearance of isolates with decreased susceptibility or resistant to third-generation cephalosporins (TGC) has raised several concerns. Recent clinical trials suggest that gentamicin could be an alternative for NG treatment, but no susceptibility data to this drug is available in European NG isolates. Objectives were: describe the epidemiological and antibiotic susceptibility profile to NG strains isolated.
Methods Descriptive retrospective study of NG strains isolated during 2011 and 2012. The susceptibility to cefixime, ceftriaxone, gentamicin and ciprofloxacin was performed using agar dilution (AD) and disc diffusion (DD) methods according to the CLSI guidelines.

Results A total of 275 NG strains were isolated: 143 urethral (52\%), 47 rectal (17\%), 44 pharyngeal (16\%), 28 endocervical (10\%), vaginal and $13(5 \%)$, belonging to 225 patients (174 men (77.3\%), 51 $(22.7 \%)$ women). The average age was 33.32 years ( $15-58$ years). About $80 \%$ of the samples (221) were from STD Clinics Infectious Service. 151 patients (67.1\%) were symptomatic at the moment of diagnosis, 32 cases (14.2\%) were detected during screening and 42 cases $(18.7 \%)$ during contact tracing. Urethritis was the most common clinical manifestation in men and accounted $71,3 \%$ of the cases.

All strains were susceptible to TGC by both methods. Ciprofloxacin showed $59.3 \%$ resistant strains by $\mathrm{AD}$ with low minor discordances between the two methods in 22 strains. $74.5 \%$ of the strains showed a MIC of $8 \mu \mathrm{g} / \mathrm{ml}$ to gentamicin (range: $2-16 \mu \mathrm{g} / \mathrm{ml}$ ), and inhibition zone in the DD method between 16 and $38 \mathrm{~mm}$.

Conclusions During the study period we found no resistant strains to TGC, but it was recorded a high rate of resistance to ciprofloxacin. The susceptibility profile of gentamicin is similar to those published but further research is needed to establish clinical breakpoints and doing a treatment recommendation.

\section{P3.281 GONORRHOEA ON THE RISE IN LATVIA - DIAGNOSTICS AND ANTIMICROBIAL RESISTANCE SURVEILLANCE}

doi:10.1136/sextrans-2013-051184.0737

'D Ozolins, ' $\mathrm{A}$ Zilevica, ${ }^{2} \mathrm{~V}$ Mavcutko, ${ }^{3} \mathrm{M}$ Unemo, ${ }^{4} \mathrm{M}$ Domeika, Eastern European Network for SexualReproductive Health. ${ }^{1}$ Faculty of Medicine, University of Latvia, Riga, Latvia; ${ }^{2}$ The Centre for Disease Prevention and Control and Infectology, Riga, Latvia; ${ }^{3}$ WHO Collaborating Centre for Gonorrhoea and other STIs, Örebro, Sweden, Orebro, Sweden; ${ }^{4}$ Department of Control and Prevention of Communicable Diseases Uppsala County Council, Uppsala, Eastern European Network for Sexual and Reproductive Health, Uppsala, Sweden

Background The emergence and international spread of antimicrobial resistance (AMR) in Neisseria gonorrhoeae has compromised the treatment and public health management of gonococcal infections. Appropriate diagnostics and surveillance of AMR are critical for controlling infections and in guiding international and national treatment guidelines to ensure appropriate patient management.

Methods A questionnaire-based survey regarding the diagnostics and AMR surveillance of N. gonorrhoeae in Latvia was conducted by the Centre for Disease Prevention and Control and Infectology of Latvia. Telephone interviews were carried out among the 73 biggest medical laboratories in Latvia.

Results In 2011, a total of 550 cases of gonorrhoea were reported giving a rate of 26.7 per 100000 population. In 2012 - 602 cases and an incidence of 29.5. In total, the incidence of gonorrhoea increased by $73.5 \%$ from 2010 to 2012 . For detection of N. gonorrhoeae, currently three laboratories are using nucleic acid amplification tests (NAATs), 10 - RNA probe hybridization tests, four laboratories culture, and 17 - Gram staining of specimen smears only. Monitoring of AMR in N. gonorrhoeae was initiated in 2010, however, during 2010 and 2012 in total only 35 isolates were tested. All 35 isolates were fully susceptible to cefixime, ceftriaxone, azithromycin, and spectinomycin, and the minimum inhibitory concentrations (MICs) of gentamicin were low. Resistance to ciprofloxacin was displayed by $43 \%$ of isolates, to penicillin by $31 \%$, and to tetracycline by $23 \%$. Conclusion The gonorrhoea incidence has significantly increased in Latvia during the recent years, which cannot only be explained by introduction of more sensitive diagnostics such as NAATs in a few laboratories. It is imperative to substantially strengthen the diagnostics and AMR surveillance in Latvia. 